\title{
MOBILE MAPPING OF THE LA CORONA LAVATUBE ON LANZAROTE
}

\author{
H. A. Lauterbach ${ }^{1, *}$, D. Borrmann ${ }^{1}$, A. Nüchter ${ }^{1}$, A.P. Rossi ${ }^{2}$, V. Unnithan ${ }^{2}$, P. Torrese ${ }^{3}$, R. Pozzobon ${ }^{4}$ \\ ${ }^{1}$ Computer Science VII - Robotics and Telematics, Julius-Maximilians-Universität Würzburg, Germany - \\ (helge.lauterbach, dorit.borrmann, andreas.nuechter)@uni-wuerzburg.de, \\ ${ }^{2}$ Physics and Earth Sciences, Jacobs University Bremen, Germany - (an.rossi, v.unnithan)@ jacobs-university.de, \\ ${ }^{3}$ Dipartimento di Scienze della Terra e dell'Ambiente, Università di Pavia, Italy - patrizio.torrese@ unipv.it, \\ ${ }^{4}$ Department of Geosciences, Università degli Studi di Padova, Italy - riccardo.pozzobon@ unipd.it
}

KEY WORDS: Mobile Mapping, Laser Scanning, SLAM, Rough Environment

\begin{abstract}
:
Planetary surfaces consist of rough terrain and cave-like environments. Future planetary exploration demands for accurate mapping. However, recent backpack mobile mapping systems are mostly tested in structured, indoor environments. This paper evaluates the use of a backpack mobile mapping system in a cave-like environment. The experiments demonstrate the abilities of an continuous-time optimization approach by mapping part of a lavatube of the La Corona volcano system on Lanzarote. We compare two strategies for trajectory estimation relying either on 2D or 3D laser scanners and show that a 3D laser scanner substantially improved the final results.
\end{abstract}

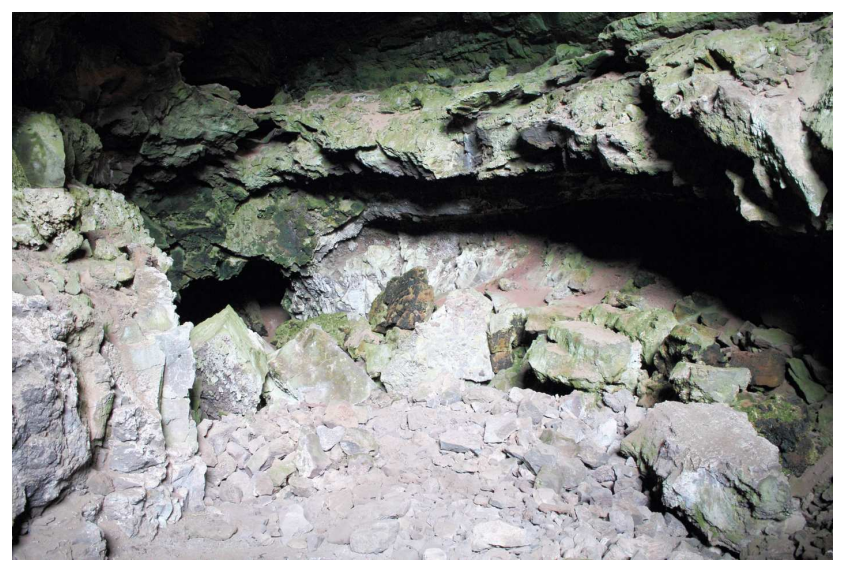

Figure 1. Photo of the La Corona lavatube on Lanzarote at the "Jameo de la Puerta Falsa"

\section{INTRODUCTION}

The exploration of Solar System bodies relies heavily on remote sensing and mapping (e.g. Massironi et al. (2018)). Planetary surface exploration, either robotic, human, or human-robotic, relies on experiments that can be tested on Earth analogues (cf. Baker (2014); Garry and Bleacher (2011). The ESA astronaut training campaign extensions PANGAEA-X (Sauro et al., 2018; Bessone et al., 2018) took place on the island of Lanzarote in 2017 and 2018 and hosted several experimental suites focussing on field geology and exploration. One of the objectives was to test technologies for exploration and mapping in low lighting conditions, lava tubes and rough terrain. Lanzarote was chosen due to its geological resemblance to Mars and Moon. One of the test suites was AGPA (Rossi et al., 2018a,b), which stands for Augmented field Geology and Geophysics for Planetary Analogues and comprises a flexible suite of remote sensing and geophysical experiments. One of the experiments conducted within AGPA is the mapping of part of the Corona lava tube, more specifically the accessible area known as "Jameo de la Puerta Falsa", shown in Figure 1.
Lidar (light detection and ranging) systems produce 3D models with high spatial accuracy independent of lighting conditions and visible features. For high precise measurements terrestrial laser scanning is the method of choice. However, in unstructured environments like the one at hand, a large amount of scanning positions are required to avoid occlusions. Mobile mapping systems with sensors mounted on cars allow for faster measurements from changing viewpoints and are the method of choice for mapping urban environments. Robotic solutions (Nüchter et al., 2013) or solutions with scanners mounted on carts, like the viametris iMMS (VIAmetris, 2015; Thomson et al., 2013), the Google Street View Trolley (Google, 2015), or the NavVis 3D Mapping Trolley (NavVis, 2015) are applicable in smaller alleys. At stairs as well as dirt or gravel roads these systems still meet their limits. Airborne laser scanning is not restricted to specific terrain and thus has advantages, but it is not available in roofed environments or tunnels or gives unsatisfying results in areas with a lot of trees or bushes. Backpack mounted systems, also known as personal laser scanning, such as "The Cartographer" by Google (Lardinois, 2015), the Zebedee 3D sensor system (Bosse et al., 2012) or the Leica Pegasus:Backpack (Leica, 2015) have been presented as ideal solutions to overcome these issues for indoor mapping. However, they have mostly been tested in structured environments.

In previous work, we presented our backpack mobile mapping system (Nüchter et al., 2015), featuring a high-end laser scanner, namely the Riegl VZ-400, for mapping with various setups and we evaluated its performance in structured indoor and outdoor environments (Lauterbach et al., 2015). In contrast to the AkhkaBackpack by Kukko et al. (2012), which also provides a high-end laser scanner, we do not incorporate a global navigation satellite system (GNSS) for localization. In urban canyons or tunnels, a GNSS-free approach is advantageous, since the GNSS signal may be disturbed, and thus, localization would fail. Corso and Zakhor (2013) and Lehtola et al. (2015) also present GNSS-free solutions based on laser scanners. However, they are currently restricted to environments where the $2.5 \mathrm{D}$ assumption holds true or to $1 \mathrm{D}$ trajectories on flat floors, respectively.

${ }^{*}$ Corresponding author 


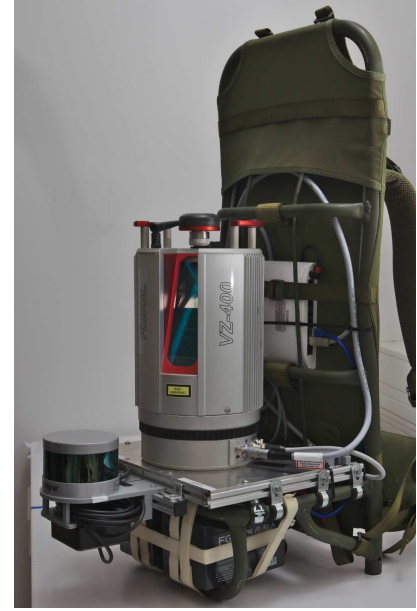

Figure 2. The backpack with Riegl VZ-400 and Velodyne VLP16 Lite laser scanners

This paper evaluates the use of the backpack mobile mapping system for use in unstructured cave-like environments where the 2.5D assumption does not hold true. Not only consists the floor of rough terrain but also the walls are highly unstructured. To enable the backpack system to work in such an environment the setup is modified. The system from (Lauterbach et al., 2015) relies on a 2D laser scanner to estimate the trajectory of the Riegl VZ400. This is shown to give poor results in unstructured environments. Therefore the 2D laser scanner is replaced by a 3D laser scanner commonly used in automotive applications improving the initial trajectory significantly. A semi-rigid SLAM (Simultaneous Localization and Mapping) approach is used to optimize the point clouds. The main contributions are the development of an upsampling-based scan registration for trajectory estimation and the improvement of a semi-rigid ICP (Iterative Closest Point) strategy.

\section{BACKPACK MOBILE MAPPING SYSTEM}

\subsection{System configuration}

The central sensor of the backpack system (cf. Fig. 2) is a Riegl VZ-400 3D laser scanner (Riegl Laser Measurement Systems, 2019). It rotates back and forth around its vertical axis, providing a horizontal field of view (FoV) of $270^{\circ}$ and a vertical FoV of $100^{\circ}$, thus continuously collecting data and avoiding the blind spot caused by the human operator. It runs at a rotational speed of $1 / 6^{\circ} \mathrm{s}^{-1}$ recording $2 \mathrm{D}$ scan lines with $120 \mathrm{~Hz}$ at an angular resolution of $0.5^{\circ}$. The maximum range is $350 \mathrm{~m}$. For motion estimation the system relies on a Velodyne VLP16 Lite 3D laser scanner. It provides full $360^{\circ}$ scans at a frequency of $10 \mathrm{~Hz}$ with a maximum range of $100 \mathrm{~m}$. The vertical FoV of $30^{\circ}$ is sparsely covered by 16 scan lines with a resolution of $2^{\circ}$ vertically and $0.2^{\circ}$ horizontally. The laser scanners are connected by LAN to a laptop running the Robot Operating System (ROS) for data logging. The offset between the two 3D sensors is calibrated by registration of laser scans in a static environment.

\subsection{Processing pipeline}

The workflow is depicted in Fig. 3. The recorded sparse 3D laser scans are upsampled and registered according to Sec. 3 to estimate the initial trajectory. Next, the 3D laser scans from the terrestrial laser scanner are extracted in the unwinding step, discretized in 2D scan slices. The initially created point cloud is then optimized with continuous-time ICP and SLAM described in Sec. 4.2 and 4.1.

\section{TRAJECTORY ESTIMATION}

Due to the flat world assumption the effectiveness of mapping systems relying on a 2D laser scanner is limited in rough environments. Nüchter et al. (2015) estimate the trajectory by processing 2D laser scans with HectorSLAM (Kohlbrecher et al., 2011). To improve results, attitude information from an inertial measurement unit (IMU) is used by HectorSLAM to reject points that are far away from the ground plane for scan matching.

Recently arising 3D laser scanners used in automotive applications combine multiple 2D scan lines at different scan angles. The resulting point clouds are sparse in vertical direction, thus traditional scan registration variants, such as the well-known ICP algorithm Besl and McKay (1992), tend to fail. This is resolved by either extracting features, that are present in multiple lines, like edges or planes (Zhang and Singh, 2014), or by making assumptions about the underlying surface. Holz and Behnke (2014) therefore create a simple quad mesh in order to estimate the point normals and then apply a variant of the Generalized-ICP (Segal et al., 2009). Similar to this we approximate the surface by upsampling the point cloud. Inspired by the idea of range images, the sensor data is first organized into a ring or bin structure, preserving the real measurement. For each point two triangles are created with its successor of the same ring and the two concurrent points in the next ring. As in (Holz and Behnke, 2014) triangles are rejected if an edge is nearly parallel to the line of sight with respect to the scan pose or a depth discontinuity is detected considering angular and range dependent thresholds. A valid triangle is then further subdivided into smaller triangles until the largest edge is shorter than a threshold. The vertices of the triangles form a virtual scan. Octree reduction generates a homogeneous distribution of points. Finally the trajectory is estimated by incremental registration using ICP and the data from the terrestrial scanner unwound using this trajectory.

\section{TRAJECTORY OPTIMIZATION}

Common 3D registration approaches expect the sensor not to move during individual measurements. As this rigidity assumption does not hold true for many applications in the automotive field or for mobile mapping, continuous-time optimization approaches have recently moved into the research focus. Two general approaches to this problem are combined for the backpack system, the optimization of the entire trajectory at once versus sequential optimization.

\subsection{Continuous-time SLAM}

Given a sufficiently estimated trajectory, the entire point cloud can be improved by optimizing the entire trajectory. We use the approach from (Elseberg et al., 2013) that is based on the ICP concept known for rigid registration algorithms. The initial point cloud is a set of scan slices, each of which is assigned a time stamped pose during the trajectory estimation. We first split the trajectory into overlapping sections and match these using the automatic high-precision registration of terrestrial 3D scans, i.e., the graph-based SLAM approach presented in (Borrmann et al., 


\section{Trajectory \\ Estimation}

Optimization
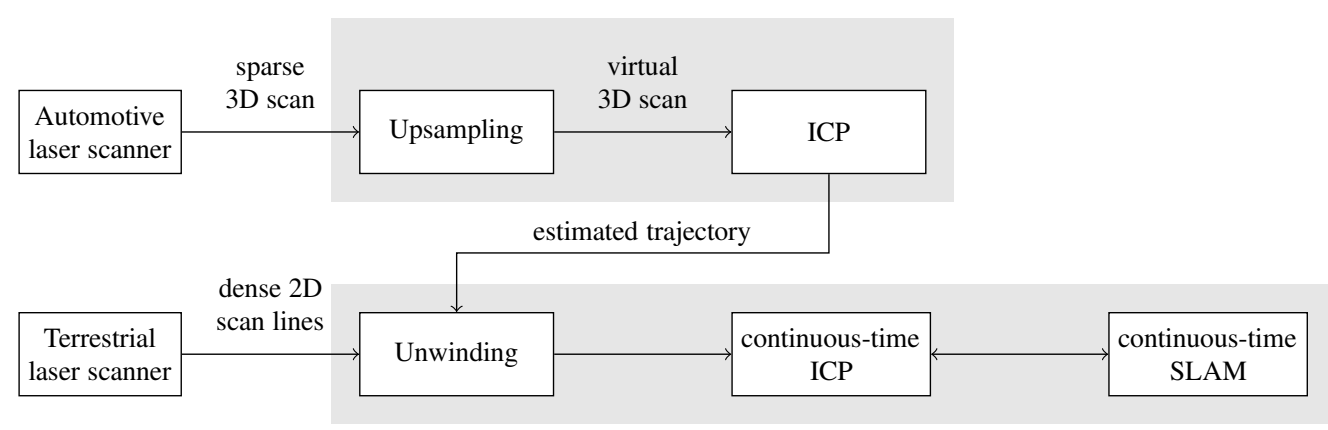

Figure 3. workflow of our backpack system

2008a). The graph is estimated using a heuristic that measures the overlap of sections based on the number of closest point pairs. After applying globally consistent scan matching on the sections the actual continuous-time or semi-rigid matching, as described in (Elseberg et al., 2013), is applied, using the results of the rigid optimization as starting values to compute the numerical minimum of the underlying least square problem. The choice of the subdivision is crucial for the results. Local trajectory errors within a sub-scan cannot be improved. A finer discretization, e.g., to single 2D scan slices, does not constrain a 6 DoF pose sufficiently.

For long trajectories in unstructured environments this global approach is problematic. If the trajectory error is larger than the features in the scene, wrong point correspondences are likely to occur and to move the point cloud into local minima. Additionally, memory requirements and runtime increase. Thus, a sequential method is developed to minimize local errors before the global optimization.

\subsection{Semi-rigid ICP}

The sequential approach of continuous-time ICP extends the work in (Elseberg et al., 2013; Lauterbach and Nüchter, 2018). To achieve separate 3D scans, as needed for scan registration, we group scan slices into submaps. The submaps are sequentially merged into larger submaps, also called meta-scans. One arbitrarily chosen scan of each submap is labeled as reference scan and defines a local coordinate system. The submaps are registered rigidly with ICP. Afterwards the transformation is locally distributed to all individual scan slices between the two reference scans. Although not constrained, a linear distribution of the translation and SLERP interpolation shows to be sufficient for small errors. Alternatively we use an error distribution based on (Elseberg et al., 2013). The ICP is applied sequentially to all submaps generated along the trajectory.

A key issue in scan registration is the search for closest point pairs. The search trees in 3DTK (Elseberg et al., 2012) are optimized for small memory footprint and fast search operations. However, due to their compact representation in memory they do not allow for dynamic extension. Thus, in sequential registration the map needs to be rebuilt for each new inserted submap. To speed up the incremental registration process, several searchtrees for already merged submaps are maintained and queried in parallel. $5 \mathrm{k}$-d trees have shown to be a good trade-off between increasing search time and reducing construction time for a new $\mathrm{k}-\mathrm{d}$ tree containing the complete meta-scan.

\section{EXPERIMENTS}

\subsection{Dataset}

The approach is tested on datasets collected during the ESA PANGAEA-X campaigns in 2017 and 2018. The test site is located in a lava tube of the La Corona volcano system on Lanzarote. This lava tube is an approximately $8 \mathrm{~km}$ long cave formed by hot lava melting rocks during its flow towards the sea. It is accessible at several so called "Jameos", where the ceiling of the tube collapsed. The experiments map an approximately $70 \mathrm{~m}$ long part of the tube at "Jameo de la Puerta Falsa". This part consists of two halls $10 \mathrm{~m}$ to $20 \mathrm{~m}$ wide and high, connected by a narrow passage of $3 \mathrm{~m}$ times $3 \mathrm{~m}$. The floor is covered by rocks of different sizes, that have to be circumvented or stepped over, as well as two steep passages of hard soil.

The experiments consist of several runs along a trajectory of $55 \mathrm{~m}$ to $60 \mathrm{~m}$ in either direction using the backpack configuration described above. Additional runs using a SICK LMS 151 2D laser profiler allow to compare the approach to the system from (Lauterbach et al., 2015). Subsequently the datasets are denoted as Trajectory3D and Trajectory2D. Table 1 summarizes the information on the presented datasets.

Terrestrial laser scans collected at 15 static positions along the trajectory (cf. Fig. 4), to minimize occlusions, serve as ground truth. These high precise and dense laser scans are registered using the ICP and SLAM (Borrmann et al., 2008b) algorithms implemented in 3DTK (Nüchter et al., 2018).

For evaluation, we apply the processing pipeline described in 2.2 to both datasets. Although continuous-time SLAM is able to use time discretization based on single points, the chosen discretization in the unwinding step results in 2D scan slices. A pose is assigned to each scan slice by interpolating linearly in time between trajectory points. Each of the iterative optimization processes that follows is repeated manually optimizing parameters such as size of the submap and maximum matching distance in a coarse to fine manner.

After applying the pipeline to each run of each dataset the data of two consecutive runs is combined into one point cloud to reduce occlusions caused by the limited FoV of the sensor. The continuous-time SLAM optimizes the trajectory. The initial trajectory for datasets using the 2D laser scanner was generated using Hector SLAM as described in (Lauterbach et al., 2015). To compensate the drift in the pose estimation from Hector SLAM and to ensure global consistency in this scenario the best results from individual optimization for each direction are selected and merged. 
Table 1. Information on the datasets presented

\begin{tabular}{c|cccccc} 
Dataset & Motion Sensor & Time $[\mathrm{s}]$ & Line Scans & Points & ICP iterations & SLAM iterations \\
\hline Trajectory3D & Velodyne VLP16 & 391 & 39937 & $40,381,128$ & $10+15$ & 200 \\
Trajectory2D & SICK LMS151 & 433 & 43019 & $35,704,888$ & 10 & $200+600+30$
\end{tabular}

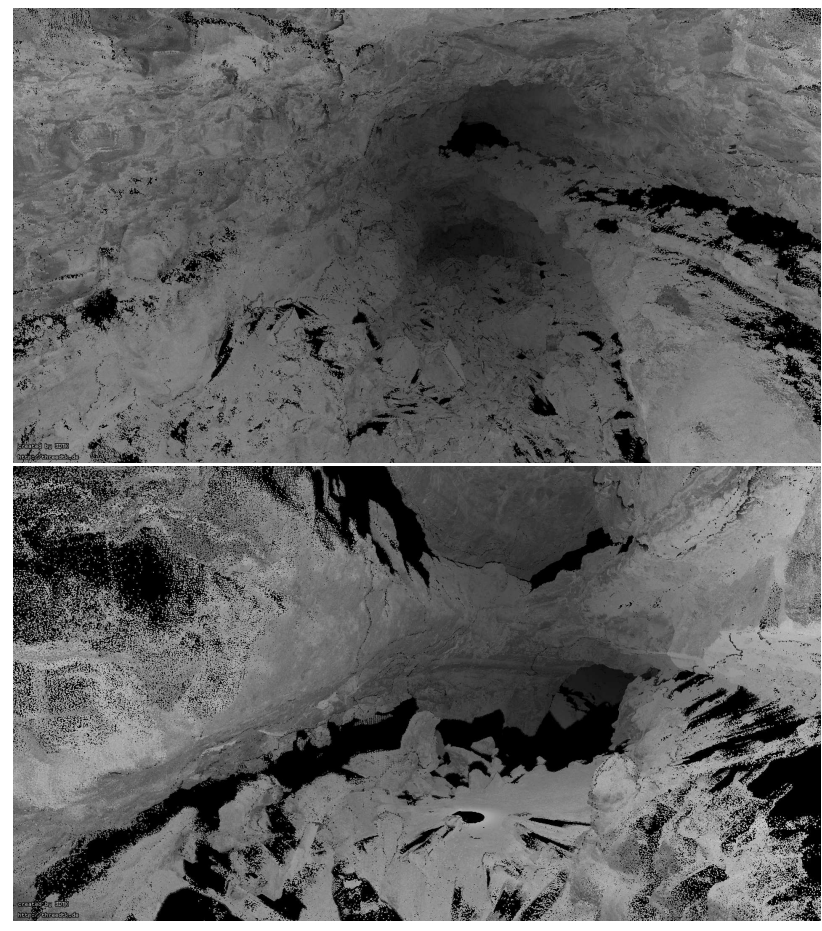

Figure 4. Reference point cloud, colored by reflectance values. Above: view near the starting point of the experiments. Below: detail at the turning point of the trajectory.

For continuous-time ICP we grouped scans of 2 or 1 revolution of the Riegl VZ-400 resulting in submaps of 960 and 480 scans, respectively. We applied 10 iterations of continuous-time ICP on each run of Trajectory3D and another 15 on the combined dataset. Continuous-time SLAM was applied only on the combined runs with 200 iterations. On Trajectory2D we apply continuous-time ICP only for 10 iterations on each run. (cf. Tab. 1) For the runs shown in Fig. 5 the continuous-time SLAM needs 600 iterations to compensate the drift in run A and 200 iterations to correct run B. Only 30 iterations on the combined dataset yield the final point cloud.

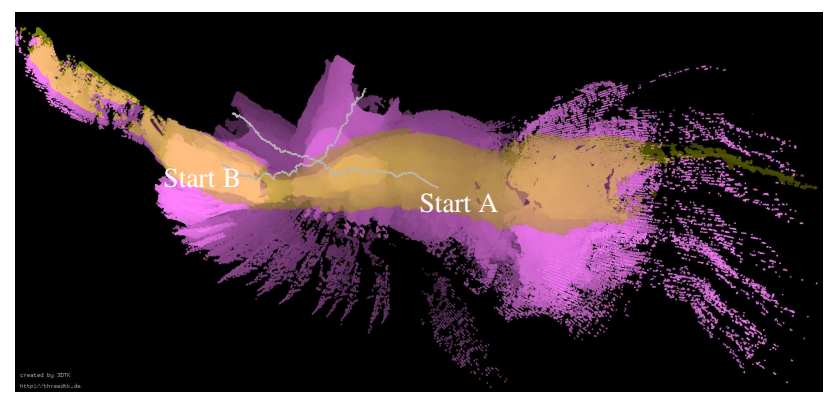

Figure 5. Unwound initial point clouds from dataset Trajectory2D w.r.t. ground truth (semi transparent yellow) from top view. The gray lines depict the estimated trajectory from HectorSLAM. At the beginning of each run A and B the point clouds match the reference cloud, but quickly diverge.
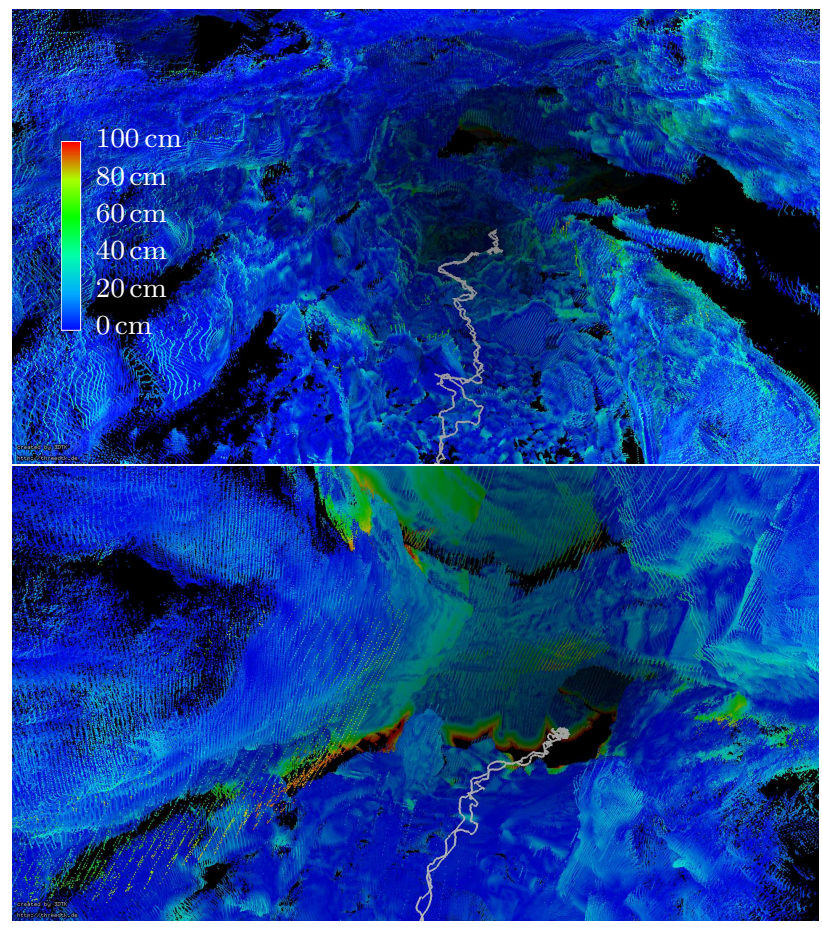

Figure 6. Final point cloud for dataset Trajectory3D, colored by deviation from ground truth. Blue indicates low and red large errors. Above: detail from the lavatube near the starting point. Below: detail at the turning point of the trajectory. The gray line resembles the trajectory.

\subsection{Results}

The estimated trajectory calculated by our approach using upsampled laser scans for registration, produces an initial point cloud that represents the environment well on a global scale. As expected the trajectories generated by HectorSLAM are prone to drift. As shown in Fig. 5, the point clouds of each run (Start $\mathrm{A}$ and $\mathrm{B}$ ) match the reference cloud near their origin but then quickly diverge. The reason for this is discussed in 3 . However, the optimization successfully corrects for this drift, so that the final point cloud of dataset Trajectory2D also reflects the general shape of the lava tube.

The cloud to cloud distance between the backpack datasets and the ground truth data gives a measure of accuracy (Fig. 6, 7, 9). Fig. 6 visualizes the deviation from ground truth for dataset Trajectory3D. Large errors are indicated in red, while points with small distance to the closest points are colored blue. The top image shows the optimized point cloud near the start respectively end of the trajectory, which corresponds to the right side of the side view in Fig. 9. To support the visual representation the corresponding error histogram in Fig. 10 shows that most errors lie in low decimeter range. $95 \%$ of all points feature an error less than $20 \mathrm{~cm}$ and even $80 \%$ an error less than $10 \mathrm{~cm}$. However the pose of some scan slices is still incorrect, thus providing errors up to $50 \mathrm{~cm}$, mainly at the walls. 

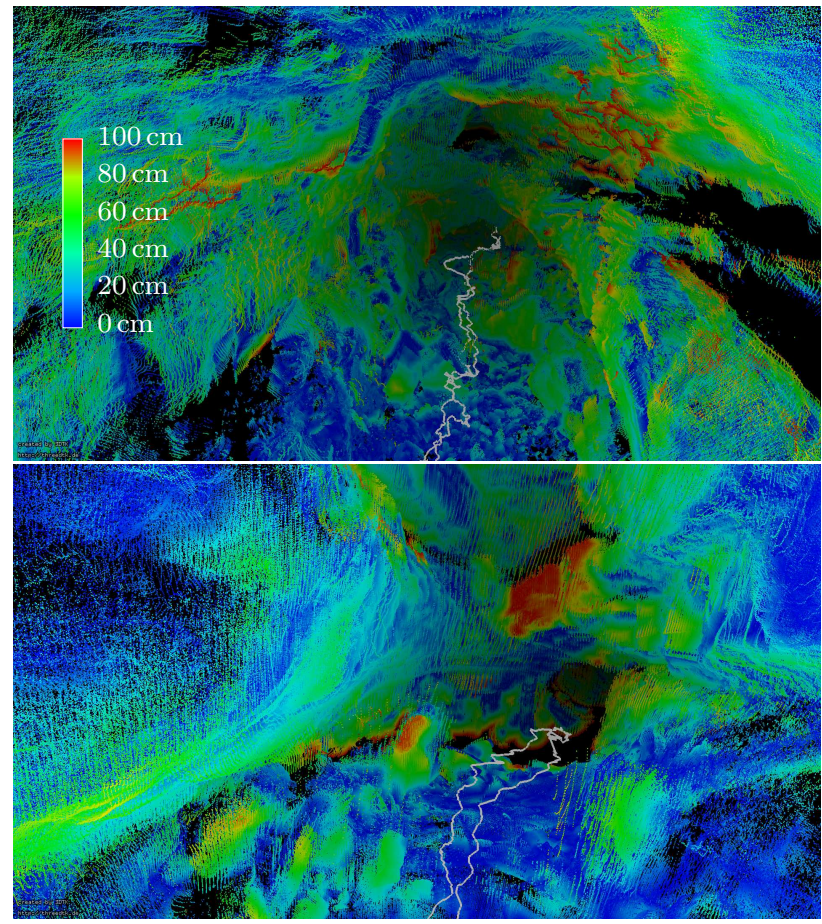

Figure 7. Final point cloud for dataset Trajectory2D, colored by deviation from ground truth. Above: detail from the lavatube near the starting point. Below: detail at the turning point of the trajectory. The gray line resembles the trajectory.

The bottom image of Fig. 6 shows the same point cloud at the turning point of the trajectory. The errors are larger in this region compared to the top image. The reasons are two-fold. First, there are gaps in the ground truth data as visualized in Fig. 8. Due to the positioning on the ground, the terrestrial laser scan (yellow) suffers from occlusions. Clearly seen by the magenta areas in the background these holes are filled by the backpack dataset Trajectory3D. As we used the distance to the closest point as an error measure, points up to the maximum search distance of $100 \mathrm{~cm}$ at the borders are wrongly assigned a high error, as seen for the green and red points in the background of Fig. 6 in the bottom image. Second, in such optimization problems the uncertainty grows with distance to the reference point, which is in our case the starting point of the trajectory. The green points at the ceiling originate from the scans near the turning point, i.e., the poses with the largest distance to the origin.

For the second dataset Fig. 7 depicts the same views as Fig. 6 does for the first dataset. As expected the error is higher in comparison to dataset Trajectory3D. The wallpoints in the top image have errors around $60 \mathrm{~cm}$ while the errors on the ceiling grow up to $100 \mathrm{~cm}$ at edges. Although individual smaller structures are still distinguishable, they appear blurred. Interestingly the errors are similarly distributed at the end of the tube (bottom image) as near the start point, except one spot on the ceiling in the middle. This becomes clear in the side view in Fig. 9. This effect originates from the fact that the starting point for the second run is near the left side and thus its trajectory is more precise near the origin. Again, the visual impression is supported by the corresponding histogram in Fig. 11. The local inaccuracy is revealed by the fact that less than $80 \%$ and $52 \%$ of all points provide an error less than $20 \mathrm{~cm}$ and $10 \mathrm{~cm}$, respectively.

To summarize the results, both approaches for trajectory estimation yield point clouds that resemble the shape of the environ-

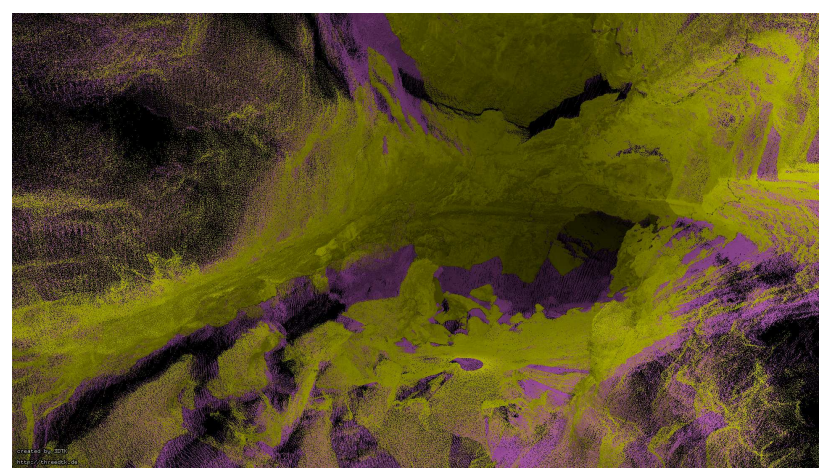

Figure 8. Comparison of dataset Trajectory3D (magenta) to ground truth (yellow). Note that the reference point cloud suffers

from occlusions that are not present in the backpack data.

ment after optimization, although the initial trajectory generated by HectorSLAM contains large drift errors. The setup with the $3 \mathrm{D}$ automotive laser scanner improves the accuracy on a local scale resulting in a point cloud with a point to point distance of less than $20 \mathrm{~cm}$ for $95 \%$ of the data.

\section{CONCLUSIONS AND FURTHER WORK}

In this paper we propose a pipeline for a backpack mobile mapping system and evaluate its use on data collected in an rough cave-like environment. In order to estimate the initial trajectory of a terrestrial laser scanner, we match sparse 3D laser scans with upsampling based registration. The applied optimization step using continuous-time ICP and SLAM manages not only to correct for large drift, but yields a point cloud, where $79 \%$ of all points have an error of less than $10 \mathrm{~cm}$.

Further work includes the application of the pipeline to open field environments as well as fusion of IMU data, that was recorded but not used in the pipeline. Open field datasets also allow for the integration of GNSS systems to further improve the trajectory estimation.

\section{ACKNOWLEDGEMENTS}

We are grateful to ESA and all PANGAEA-X staff for support in the field and to Riku Koski for his help with registering the ground truth data set. We acknowledge Matthias Maurer for his participation in the data acquisition during some of the experiments.

\section{References}

Baker, V. R., 2014. Terrestrial analogs, planetary geology, and the nature of geological reasoning. Planetary and Space Science 95, pp. 5 - 10. Planetary Geology Field Symposium, Kitakyushu, Japan, 2011: Planetary Geology and Terrestrial Analogs.

Besl, P. and McKay, N., 1992. A Method for Registration of 3-D Shapes. IEEE Transactions on Pattern Analysis and Machine Intelligence 14(2), pp. $239-256$.

Bessone, L., Sauro, F., Maurer, M. and Piens, M., 2018. Testing technologies and operational concepts for field geology exploration of the Moon and beyond: the ESA PANGAEA-X campaign. In: Geophysical Research Abstracts, Vol. 20, Vienna, Austria. EGU2018-4013. 

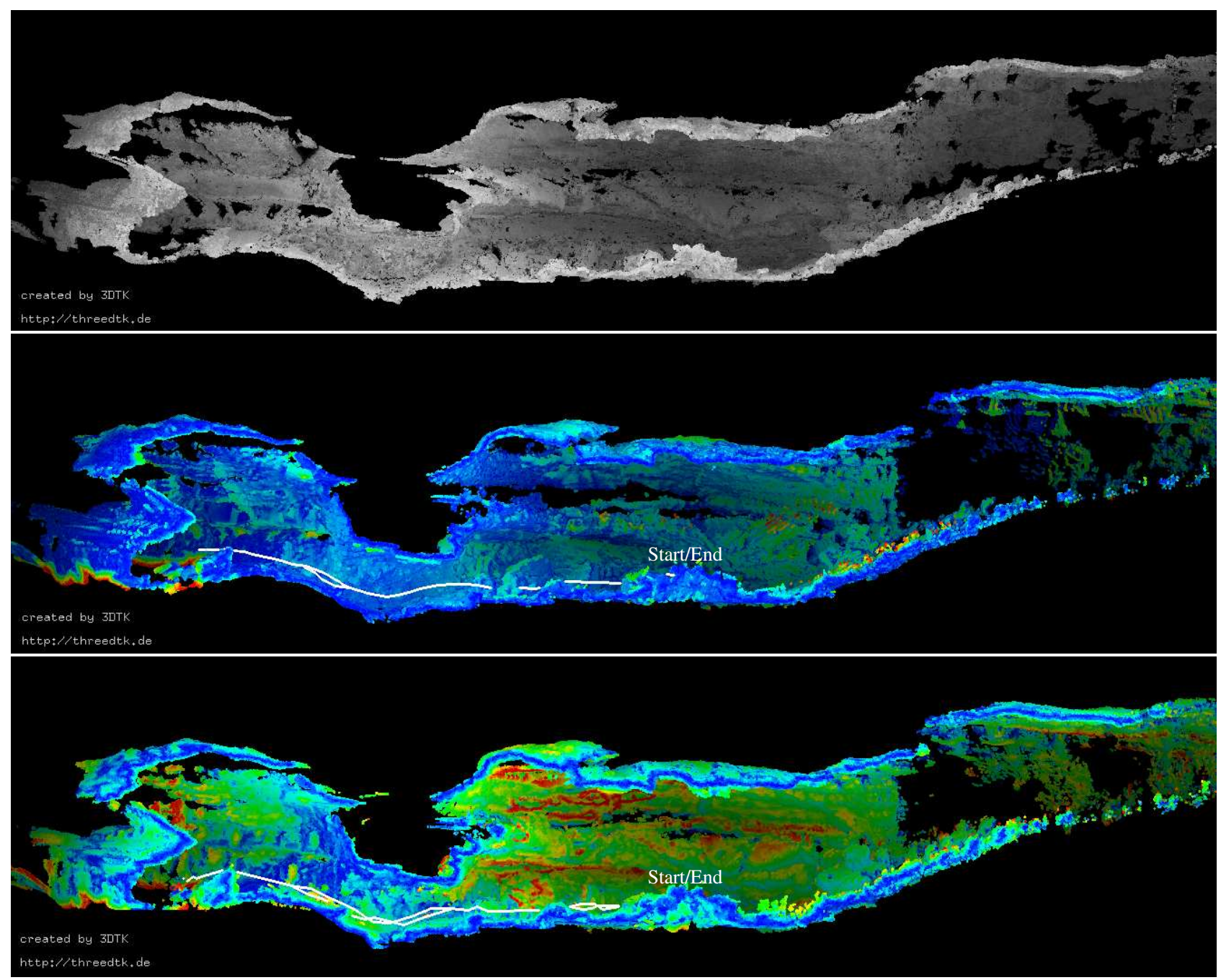

Figure 9. Side view of the point clouds. The front plane is cropped for better visibility. Above: Ground truth. Middle: Dataset Trajectory3D. Below: Dataset Trajectory2D. Ground truth is colored by reflectance values. Dataset Trajectory3D and dataset Trajectory $2 \mathrm{D}$ are colored by deviation from ground truth. Blue indicates low and red large errors.

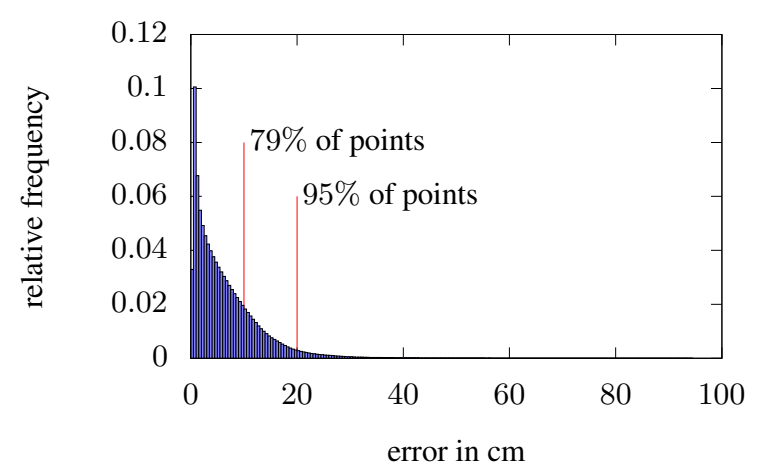

Figure 10. Deviation of dataset Trajectory3D from ground truth.

Borrmann, D., Elseberg, J., Lingemann, K., Nüchter, A. and Hertzberg, J., 2008a. Globally consistent 3d mapping with scan matching. Journal Robotics and Autonomous Systems (JRAS) 56(2), pp. 130-142.

Borrmann, D., Elseberg, J., Lingemann, K., Nüchter, A. and Hertzberg, J., 2008b. The Efficient Extension of Globally Consistent Scan Matching to 6 DoF. In: Proceedings of the 4th In-

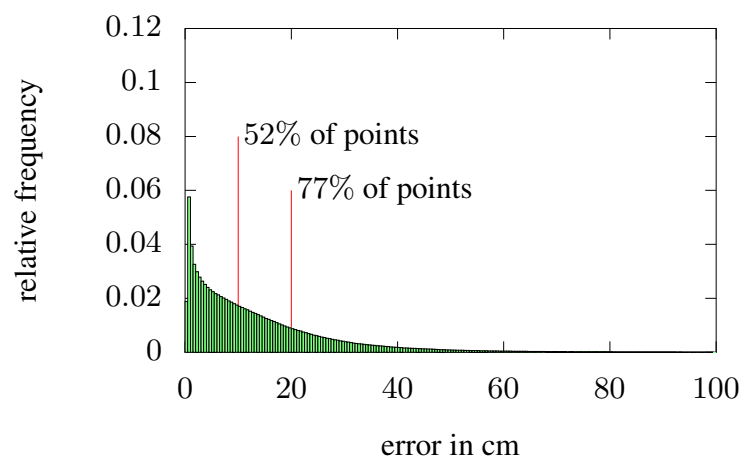

Figure 11. Deviation of dataset Trajectory2D from ground truth.

ternational Symposium on 3D Data Processing, Visualization and Transmission (3DPVT '08), Atlanta, USA, pp. 29-36.

Bosse, M., Zlot, R. and Flick, P., 2012. Zebedee: Design of a spring-mounted 3-d range sensor with application to mobile mapping. Robotics, IEEE Transactions on 28(5), pp. 11041119.

Corso, N. and Zakhor, A., 2013. Indoor localization algorithms 
for an ambulatory human operated $3 \mathrm{~d}$ mobile mapping system. Remote Sensing 5(12), pp. 6611-6646.

Elseberg, J., Borrmann, D. and Nüchter, A., 2013. Algorithmic solutions for computing accurate maximum likelihood 3D point clouds from mobile laser scanning platforms. Remote Sensing 5(11), pp. 5871-5906.

Elseberg, J., Magnenat, S., Siegwart, R. and Nüchter, A., 2012. Comparison on nearest-neigbour-search strategies and implementations for efficient shape registration. Journal of Software Engineering for Robotics (JOSER) 3(1), pp. 2-12.

Garry, W. B. and Bleacher, J. E., 2011. Analogs for Planetary Exploration. Geological Society of America.

Google, 2015. Street View Trolley. http://maps . google.com/intl/en/maps/about/behind-thescenes/streetview.

Holz, D. and Behnke, S., 2014. Registration of non-uniform density $3 \mathrm{~d}$ point clouds using approximate surface reconstruction. In: Proc. of the 45th Int. Symposium on Robotics (ISR) and 8th German Conference on Robotics (ROBOTIK).

Kohlbrecher, S., Von Stryk, O., Meyer, J. and Klingauf, U., 2011. A flexible and scalable slam system with full $3 \mathrm{~d}$ motion estimation. In: Safety, Security, and Rescue Robotics (SSRR), 2011 IEEE International Symposium on, IEEE, pp. 155-160.

Kukko, A., Kaartinen, H., Hyyppä, J. and Chen, Y., 2012. Multiplatform mobile laser scanning: Usability and performance. Sensors 12(9), pp. 11712-11733.

Lardinois, F., 2015. Google Unveils The Cartographer, Its Indoor Mapping Backpack. http://techcrunch.com/2014/09/ 04/google-unveils-the-cartographer-its-indoormapping-backpack/.

Lauterbach, H. A. and Nüchter, A., 2018. Preliminary Results on Instantaneous UAV-Based 3D Mapping for Rescue Applications. In: Proceedings of the 16th IEEE International Symposium on Safety, Security, and Rescue Robotics (SSRR'18), Philadelphia, PA, USA, pp. 1-2.

Lauterbach, H. A., Borrmann, D., Hess, R., Eck, D., Schilling, K. and Nüchter, A., 2015. Evaluation of a backpack-mounted 3d mobile scanning system. Remote Sensing 7(10), pp. 1375313781.

Lehtola, V. V., Virtanen, J.-P., Kukko, A., Kaartinen, H. and Hyyppä, H., 2015. Localization of mobile laser scanner using classical mechanics. ISPRS Journal of Photogrammetry and Remote Sensing 99, pp. 25-29.

Leica, 2015. Leica pegasus:backpack. www.leicageosystems.com/de/Leica-PegasusBackpack_106730. htm.

Massironi, M., Altieri, F., Hiesinger, H., Mangold, N., Rothery, D., Rossi, A. P., Balme, M., Carli, C., Capaccioni, F., Cremonese, G., Filacchione, G., Mouelic, S. L., Unnithan, V. and Bogert, C. V. D., 2018. Towards integrated geological maps and 3D geo-models of planetary surfaces: the H2020 PLANetary MAPping project. In: Geophysical Research Abstracts, Vol. 20, Vienna, Austria. EGU2018-18106.

NavVis, 2015. M3 trolley. https://www.navvis.com/ explore/trolley/.

Nüchter, A., Borrmann, D., Elseberg, J. and Redondo, D., 2015. A Backpack-Mounted 3D Mobile Scanning System. Allgemeine Vermessungs-Nachrichten (AVN), Special Issue MoLAS 2014 122(10), pp. 301-307.
Nüchter, A., Elseberg, J. and Borrmann, D., 2013. Irma3D - An Intelligent Robot for Mapping Applications. In: Proceedings of the 3rd IFAC Symposium on Telematics Applications (TA '13), Seoul, Korea, pp. 119-124.

Nüchter et al., 2018. 3DTK - The 3D Toolkit. http:// threedtk.de/.

Riegl Laser Measurement Systems, 2019. Data Sheet, Riegl VZ-400. http://www.riegl.com/uploads/tx pxpriegldownloads/10_DataSheet_VZ-400_2017-0614.pdf.

Rossi, A. P., Unnithan, V., Torrese, P., Borrmann, D., Nüchter, A., Lauterbach, H., Ortenzi, G., Jährig, T. and Sohl, F., 2018a. Augmented field Geology and Geophysics for Planetary Analogues. In: Proceedings of the EGU General Assembly 2018, Pico session: Planetary geobiological analogs for Mars and beyond: Field, lab and simulations, Vol. 20, Vienna, Austria. EGU2018-6389.

Rossi, A. P., Unnithan, V., Torrese, P., Borrmann, D., Nüchter, A., Lauterbach, H., Ortenzi, G., Jährig, T., Sohl, F., Pozzobon, R. and Minin, M., 2018b. APGA: Integrating field Geology and Geophysics for Planetary Analogues. In: European Planetary Science Congress (EPSC 2018), Vol. 12. EGU2018-408.

Sauro, F., Massironi, M., Pozzobon, R., Hiesinger, H., Mangold, N., Martinez-Frias, J., Cockell, C. and Bessone, L., 2018. Training astronauts for field geology: the ESA PANGAEA training and PANGAEA-eXtension testing analogue. In: LPSC $X L I X$.

Segal, A., Hähnel, D. and Thrun, S., 2009. Generalized-icp. In: Proceedings of Robotics: Science and Systems.

Thomson, C., Apostolopoulos, G., Backes, D. and Boehm, J., 2013. Mobile laser scanning for indoor modelling. ISPRS Annals of Photogrammetry, Remote Sensing and Spatial Information Sciences II-5/W2, pp. 289-293.

VIAmetris, 2015. Mobile Mapping Technology. http://www . viametris.com/.

Zhang, J. and Singh, S., 2014. Loam: Lidar odometry and mapping in real-time. In: Robotics: Science and Systems, Vol. 2, p. 9. 